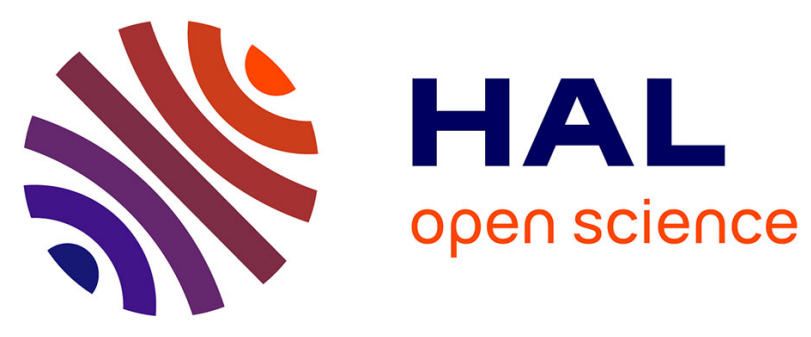

\title{
Advances and Promises of Layered Halide Hybrid Perovskite Semiconductors
}

\author{
Laurent Pedesseau, Daniel Sapori, Boubacar Traoré, Roberto Robles, \\ Hong-Hua Fang, Maria Antonietta Loi, Hsinhan Tsai, Wanyi Nie, \\ Jean-Christophe Blancon, Amanda J Neukirch, et al.
}

\section{To cite this version:}

Laurent Pedesseau, Daniel Sapori, Boubacar Traoré, Roberto Robles, Hong-Hua Fang, et al.. Advances and Promises of Layered Halide Hybrid Perovskite Semiconductors. ACS Nano, 2016, 10 (11), pp.97769786. 10.1021/acsnano.6b05944 . hal-01384468

\section{HAL Id: hal-01384468 https://hal.science/hal-01384468}

Submitted on 14 Dec 2016

HAL is a multi-disciplinary open access archive for the deposit and dissemination of scientific research documents, whether they are published or not. The documents may come from teaching and research institutions in France or abroad, or from public or private research centers.
L'archive ouverte pluridisciplinaire HAL, est destinée au dépôt et à la diffusion de documents scientifiques de niveau recherche, publiés ou non, émanant des établissements d'enseignement et de recherche français ou étrangers, des laboratoires publics ou privés. 


\title{
Advances and Promises of Layered Halide Hybrid Perovskite Semiconductors
}

\author{
Laurent Pedesseau ${ }^{\dagger}$ Daniel Sapori, ${ }^{\ddagger}$ Boubacar Traore, ${ }^{\ddagger}$ Roberto Robles,, \\ Hong-Hua Fang, $§$ Maria A. Loi, ${ }^{\S}$ Hsinhan Tsai, "Wanyi Nie, I Jean-Christophe \\ Blancon," Amanda Neukirch," Sergei Tretiak," Aditya D. Mohite," Claudine \\ Katan, ${ }^{\ddagger}$ Jacky Even, ${ }^{*, \dagger}$ and Mikaël Kepenekian ${ }^{*, \dagger}$
}

Fonctions Optiques pour les TélécommunicatiONs (FOTON), INSA de Rennes, CNRS, UMR 6082, 35708 Rennes, France, Institut des Sciences Chimiques de Rennes (ISCR), Université de Rennes 1, CNRS, UMR 6226, 35042 Rennes, France, ICN2 - Institut Catala de Nanociencia i Nanotecnologia, Campus UAB, 08193 Bellaterra (Barcelona), Spain, Zernike Institute for Advanced Materials, University of Groningen, Nijenborgh 4, Groningen, 9747 AG, The Netherlands, and Los Alamos National Laboratory, Los Alamos, NM 87545, U.S.A.

E-mail: jacky.even@insa-rennes.fr; mikael.kepenekian@univ-rennes1.fr

\footnotetext{
${ }^{*}$ To whom correspondence should be addressed

${ }^{\dagger}$ Fonctions Optiques pour les TélécommunicatiONs (FOTON), INSA de Rennes, CNRS, UMR 6082, 35708 Rennes, France

${ }^{\ddagger}$ Institut des Sciences Chimiques de Rennes (ISCR), Université de Rennes 1, CNRS, UMR 6226, 35042 Rennes, France

IICN2 - Institut Catala de Nanociencia i Nanotecnologia, Campus UAB, 08193 Bellaterra (Barcelona), Spain

${ }^{\S}$ Zernike Institute for Advanced Materials, University of Groningen, Nijenborgh 4, Groningen, 9747 AG, The Netherlands

"Los Alamos National Laboratory, Los Alamos, NM 87545, U.S.A.
} 


\begin{abstract}
Layered halide hybrid organic-inorganic perovskites (HOP) have been the subject of intense investigation before the rise of 3-dimensional (3D) HOP and their impressive performance in solar cells. Recently, layered HOP have also been proposed as attractive alternatives for photostable solar cells, and revisited for light emitting devices. In this review, we combine classical solid-state physics concepts with simulation tools based on density functional theory (DFT) to overview the main features of the optoelectronic properties of layered HOP. A detailed comparison between layered and 3D HOP is performed to highlight differences and similarities. In the same way as the cubic phase was established for 3D HOP, here we introduce the tetragonal phase with $D_{4 h}$ symmetry as the reference phase for 2D layered HOP. It allows for detailed analysis of the spinorbit coupling effects and structural transitions with corresponding electronic band folding. We further investigate the effects of octahedral tilting on the band-gap, loss of inversion symmetry and possible Rashba effect, quantum confinement and dielectric confinement related to the organic barrier, up to excitonic properties. Altogether this paper aims to provide an interpretive and predictive framework for $3 \mathrm{D}$ and $2 \mathrm{D}$ layered HOP optoelectronic properties.
\end{abstract}

\title{
Table of Contents Graphical Abstract
}

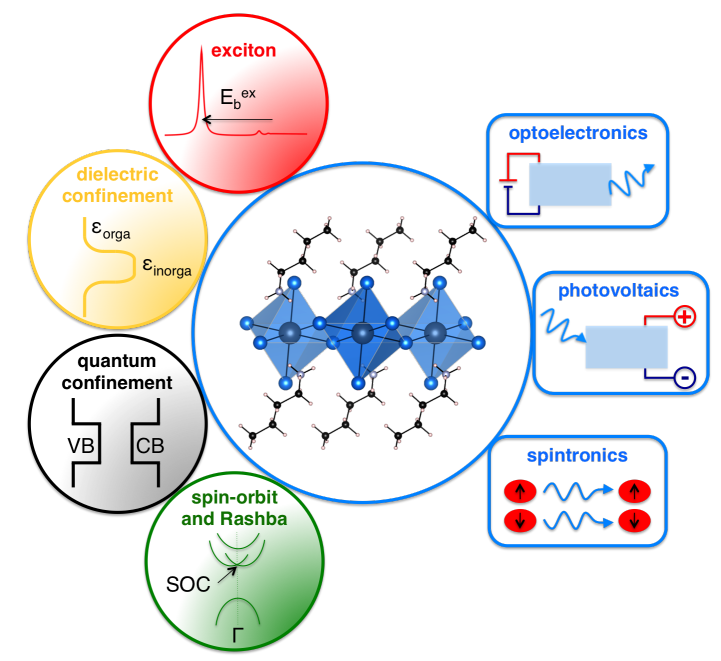




\section{Introduction}

After 2012, following the first convincing results in light-to-electricity conversion, ${ }^{1-8} 3$ dimensional (3D) halide hybrid organic-inorganic perovskites (HOP) have gained a key position in the solar light-harvesting applications. These materials have general formula $\mathrm{AMX}_{3}$, where $\mathrm{X}, \mathrm{M}$, and $\mathrm{A}$ are a halogen, a metallic cation $\left(\right.$ e.g. $\left.\mathrm{Pb}^{2+}\right)$ and a 'small' organic cation (e.g. $\mathrm{CH}_{3} \mathrm{NH}_{3}^{+}$), respectively. Since then, HOP based solar cells have shown growing conversion efficiencies. At the time we are concluding this review, the latest certified records exceed 21\%. ${ }^{9-11}$ Importantly, excellent efficiencies have been obtained recently for cells with larger areas: $15.6 \%$ and $19.6 \%$ for a $1 \mathrm{~cm}^{2}$ cell ${ }^{12,13}$ and $10.4 \%$ for $10.1 \mathrm{~cm}^{2} .{ }^{14}$ Combined with low-cost production and easy material synthesis, such performances place these newly born solar cells among the most promising technologies for the future. Putting aside the issue of lead toxicity, ${ }^{15-17}$ the last obstacle on the path of HOP solar cells appears to be their instability to air and light exposure. ${ }^{18,19}$

The next evolution of HOP might come from their past with the revival of layered, i.e. 2-dimensional (2D), HOP structures and mixed 2D/3D structures. Indeed, due to extended chemical engineering possibilities, attractive light absorption and emission, and remarkable electronic properties at room temperature, layered HOP have increased the interest in the field of optoelectronics and microelectronics for over 30 years. ${ }^{20-30}$ Despite lower dimensional systems having long been studied, they might also be the future of HOP-based technologies with revitalized research efforts on layered systems, including colloidal materials. ${ }^{31-37}$ In particular, 2D and 2D/3D materials are now exhibiting improved stability and growing performances in photovoltaic devices. ${ }^{31,32,38}$

Theoretical studies have closely paralleled the advancements of 3D HOP solar cells. ${ }^{39-42}$ Most theoretical contributions rely on the use of density functional theory (DFT) based simulations. ${ }^{43,44}$ Some of us have contributed to this effort and have shown that such atomistic calculations can be efficiently combined with complementary approaches: semi-empirical models based on symmetry considerations, basic solid-state physics concepts, Landau the- 
ory of phase transitions as well as accurate approaches to the stochastic molecular motions, which were initially developed for hybrid plastic crystals. ${ }^{45-49}$ Noteworthy, in 3D HOP thermally activated disorder related to small organic cations is a major issue for quantitative predictions of electronic properties at room temperature using standard DFT codes. ${ }^{48,49}$ At low temperature, orientations of the cation are expected to be frozen and DFT calculations can be more predictive, but at the price of a larger number of atoms per unit cell. ${ }^{39-42}$ In contrast, the theoretical models relevant to layered HOP are much less developed. Due to very large size of the unit cells of 2D HOP structures, DFT calculations are often very limited by available computational resources. For instance, state-of-the-art DFT computations including many-body effects or DFT resolution of the Bethe Salpeter equation ${ }^{50}$ (BSE) for the excitonic effects are beyond our reach. Such limitations of first-principles based theoretical approaches are particularly detrimental, and the modeling of layered HOP remains crude and highly empirical, while their potential for technological applications is immensely broad. The dielectric mismatch between the inorganic and organic layers is expected to play a major role for optical properties and charge transport, as well as to provide a natural protection against photodegradation or moisture. ${ }^{38} \mathrm{~A}$ computational modeling at the atomic scale of both layers including the interface, is thus important.

In this review, we demonstrate that concepts and theoretical tools already well deployed in the fields of optoelectronics and conventional semiconductors, and more recently in 3D HOP, are transferrable means to the investigation of layered HOP as well. First and foremost, we scrutinize basic properties of the electronic structure related to reverse band ordering, as compared to conventional semiconductors, spin-orbit coupling (SOC) and quantum confinement. We then introduce a reference structure for 2D HOP, define the corresponding Brillouin zone (BZ) folding and illustrate related effects on real layered structures. Subsequently, structural phase transitions and related distortions of the inorganic lattice, as well as their effect on the electronic band gap are examined. The possibility of symmetry inversion loss and related Rashba effect are also discussed. Finally, before concluding remarks, 
dielectric confinement in layered HOP is thoroughly investigated based on a recently developed nanoscale description. This introduces an alternative framework to compute excitonic effects based on the resolution of the BSE, which is currently unfeasible at the DFT level mainly because of the unit cell sizes.

\section{Reverse band ordering, spin-orbit coupling and quantum confine- ment}

A common feature of 3D and layered lead-based HOP is the strong SOC effect on the conduction band $(\mathrm{CB})$ due to the dominant role of the metallic $p$ orbitals of $\mathrm{Pb}{ }^{27,39}$ Notably, HOP present a reversed effect as compared to conventional semiconductors where the stronger manifestation of SOC is observed in the valence band (VB). As expected from tabulated atomic splittings, the coupling is stronger with $\mathrm{Pb}^{2+}$ (Figure 1-c) and lesser with $\mathrm{Sn}^{2+}$ (Figure 1-d). ${ }^{27,46}$ The same trend is observed in Table 1 which reports band gaps for various layered compounds including series $\left(\mathrm{Bu}-\mathrm{NH}_{3}\right)_{2} \mathrm{MI}_{4}(\mathrm{M}=\mathrm{Pb}, \mathrm{Sn}, \mathrm{Ge})$. Surprisingly, the Ge system still presents a sizable band gap reduction of $0.15 \mathrm{eV}$ with SOC. The same effect was predicted for the $\operatorname{Pm} \overline{3} m$ phase of tri-chloride based Ge 3D HOP and attributed to the SOC splitting between the $p$-like orbitals of total angular momentum $\mathrm{J}=3 / 2$ and $\mathrm{J}=1 / 2$ which amounts to $0.22 \mathrm{eV}$ for $\mathrm{Ge}^{2+} .{ }^{46}$

The main difference between layered and 3D HOP is that the CB Minimum (CBM) is only composed from two $p$-like orbitals in 2D HOP instead of three orbitals in 3D ones. Shear strain effects are inherent to layered HOP, leading to a weak degeneracy lifting and to energy minima slightly shifted away from $\Gamma .{ }^{27}$ However, this effect is dominated by SOC, and the energy minima are retrieved exactly at the $\Gamma$-point for both split states, leading to pure direct electronic transitions (Figure 1-c and -d). A dramatic consequence of SOC in $\mathrm{HOP}$ is the DOS reduction in the $\mathrm{CB}$ by a factor of 3 in 3D materials, and a factor of 2 in 2D compounds. ${ }^{27,39}$ The DOS shrinking deeply affects both transport and optical properties. For example, optical absorptions of $3 \mathrm{D}$ and $2 \mathrm{D}$ materials are reduced by the same factors 3 
Table 1: Calculated electronic band gaps $E_{g}(\mathrm{eV})$, axial (out-of-plane) $d_{M-I}^{a x}$ and equatorial (inplane) $d_{M-I}^{e q}$ distances $(\AA)$ and equatorial (in-plane) angles M-I-M $(\mathrm{M}=\mathrm{Pb}, \mathrm{Sn}, \mathrm{Ge})$ for $(p \mathrm{FPhEt}-$ $\left.\mathrm{NH}_{3}\right)_{2} \mathrm{PbI}_{4},{ }^{51,52}\left(p \mathrm{FPhEt}-\mathrm{NH}_{3}\right)_{2} \mathrm{SnI}_{4},{ }^{25}\left(\mathrm{Bu}-\mathrm{NH}_{3}\right)_{2} \mathrm{PbI}_{4},{ }^{53}\left(\mathrm{Bu}-\mathrm{NH}_{3}\right)_{2} \mathrm{SnI}_{4},{ }^{54}\left(\mathrm{Bu}-\mathrm{NH}_{3}\right)_{2} \mathrm{GeI}_{4},{ }^{55}$ $\left(\text { Pentyl- }-\mathrm{NH}_{3}\right)_{2} \mathrm{PbI}_{4}{ }^{53}$ and $\left(\text { Decyl- } \mathrm{NH}_{3}\right)_{2} \mathrm{PbI}_{4} \cdot{ }^{56}$

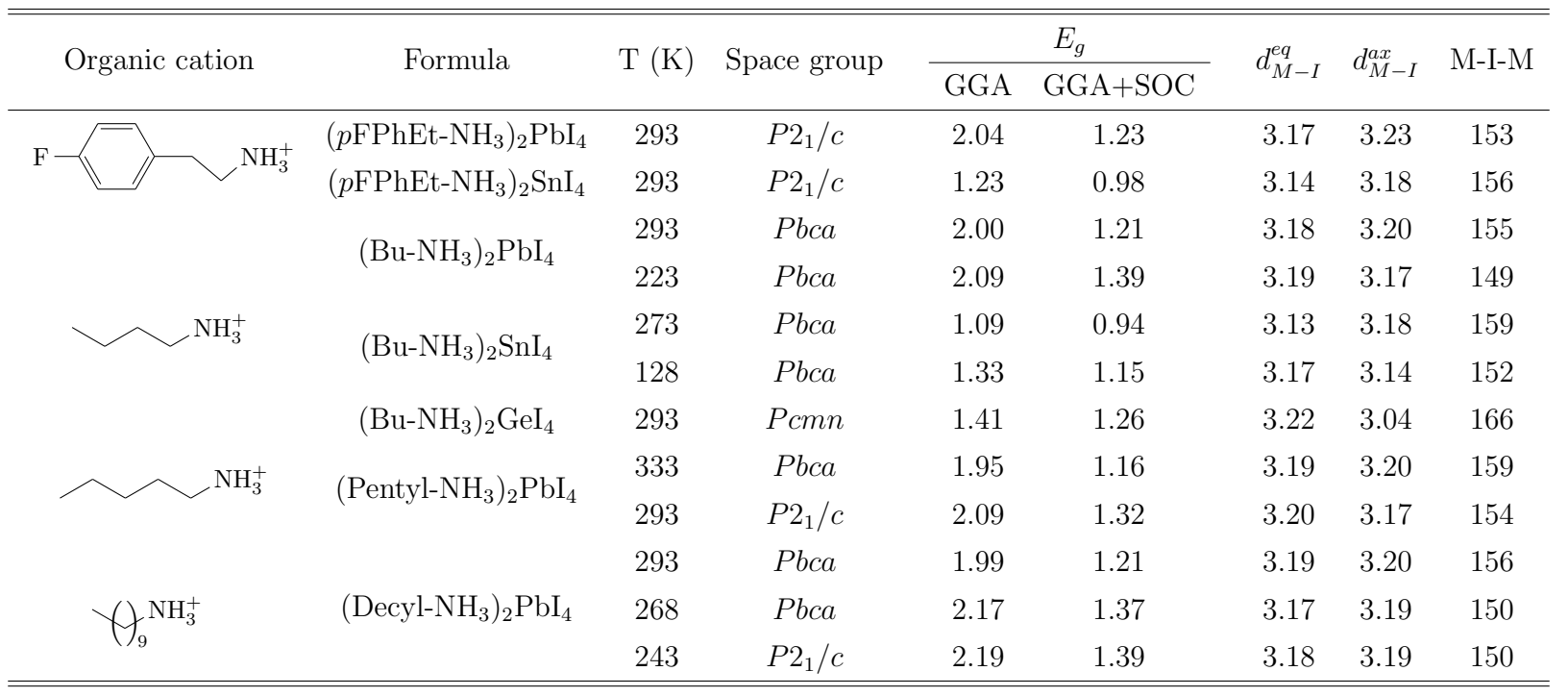

and 2, respectively. ${ }^{27}$ Fortunately, since both conduction and valence bands are associated with dispersion parabola with almost the same effective masses (i.e. band curvatures), a well-balanced in-plane carrier transport is predicted for layered HOP. ${ }^{27}$

In a pioneer contribution, Mitzi and coworkers proposed to consider layered HOP as type I quantum wells (QW) built from semiconducting inorganic sheets electronically confined by organic layers with much wider band gaps. ${ }^{25}$ Unfortunately, this elegant representation cannot be easily transposed from the classical semiconductors to 2D HOP. Typical systems, such as para-fluorophenethylammonium based layered HOP $\left(p \text { FPhEt- } \mathrm{NH}_{3}\right)_{2} \mathrm{MI}_{4}(\mathrm{M}=\mathrm{Pb}$, Sn, Figure 1-a and -b) present band structures with a direct character at the $\Gamma$-point (Figure 1-c and $-\mathrm{d}$ ) and no dispersion along the $\Gamma-\mathrm{X}$ direction (characterizing the stacking axis in real space), which are consistent with a type I QW. These features signify of a density of states (DOS) with reduced 2D dimensionality close to the band gap. ${ }^{26-29}$ However, while defining the A/B heterostructure (i.e. the layered HOP) is straightforward, the corresponding $\mathrm{A}$ and $\mathrm{B}$ bulk materials need to be delineated with caution. For instance, DFT can 
afford quantitatively assess the CB and VB alignments but the 2D HOP structure must be described as a composite material. ${ }^{28,47}$

More importantly, the type I QW-like picture cannot be associated with an effective mass model to evaluate quantum confinement effects. This model predicts superlattice effects which are neither observed experimentally nor appear in DFT simulations. Another fundamental limitation of such an empirical approach comes from the computed energies of the confined charge carriers in the inorganic lattice. Those are taken from the reference 3D bulk $\mathrm{CH}_{3} \mathrm{NH}_{3} \mathrm{MX}_{3}(\mathrm{M}=\mathrm{Pb}, \mathrm{Sn}$, and $\mathrm{X}=\mathrm{I}, \mathrm{Br}, \mathrm{Cl})$ but lie in a range of strong nonparabolicity. Last, in a classical semiconductor heterostructure built with various materials, effective mass model requires common Bloch basis functions. This requirement is clearly not satisfied in the case of layered HOP. ${ }^{28}$

Therefore, although very deep confinement potentials are well described and simulated, they cannot be coupled with effective mass modeling to accurately investigate quantum confinement effects. Subsequently, layered HOP must be considered as real composite materials rather than semiconductor superlattices. The discussion on quantum confinement can be carried out for HOP presenting even lower dimensions. For instance, a layered HOP with both face and corner-sharing octahedra has recently demonstrated additional confinement in one direction of the inorganic sheet due to its particular structure. ${ }^{57}$

\section{Brillouin zone folding from reference phases to distorted structures}

Structural, electronic and optoelectronic properties of 3D HOP can be understood by considering the pseudo-cubic $P m \overline{3} m$ perovskite phase as a reference phase. ${ }^{39,47,48}$ In particular, the band structure of the Pnma and $I 4 / \mathrm{mcm}$ phases of the most popular $\mathrm{HOP}, \mathrm{CH}_{3} \mathrm{NH}_{3} \mathrm{PbI}_{3}$, are easily interpreted starting from $P m \overline{3} m$. If the electronic band structures of the Pnma and $I 4 / \mathrm{mcm}$ phases seem very different from that of the reference $P m \overline{3} m$ phase, plotting the band structure of the latter using the BZ of the target phases reveals the opposite. Apart from small perturbations related to an increase of the electronic band gap and a small 
(a)

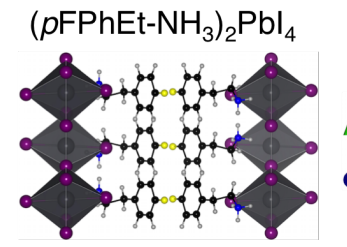

(c)

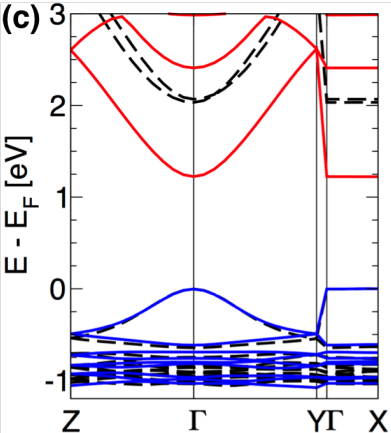

(b)

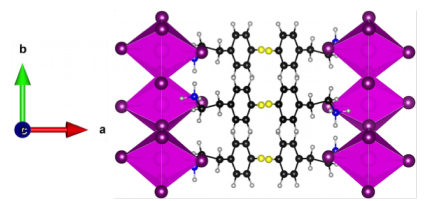

(d) 3

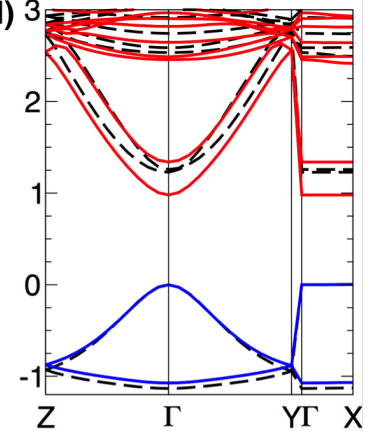

(e) $\quad D_{4 h}$ simple cell
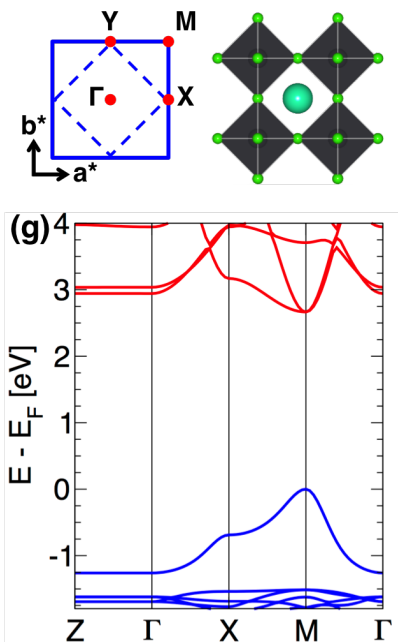

(f)

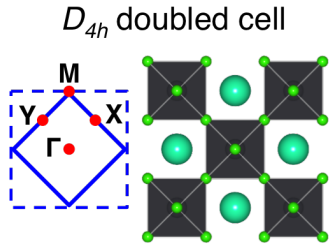

(h) 4

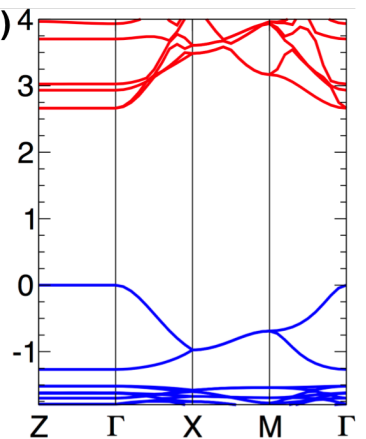

Figure 1: (a), (b) Structures and (c), (d) DFT band structures of $\left.(p \text { FPhEt-NH})_{2}\right)_{2 b I}$ and $\left(p \text { FPhEt- } \mathrm{NH}_{3}\right)_{2} \mathrm{SnI}_{4}$, respectively, computed with PBE without (blue dashed line) and with (red solid line) SOC. The zero-energy is referenced to the valence band maximum. $\mathrm{Pb}, \mathrm{Sn}, \mathrm{I}, \mathrm{C}, \mathrm{F}$, and $\mathrm{H}$ atoms are depicted in grey, pink, purple, black, yellow and white, respectively. (e) Artificially designed highly symmetric $D_{4 h}$ layered structure of $(\mathrm{Cs})_{2} \mathrm{PbCl}_{4}$ and the corresponding Brillouin zone (BZ). (f) The same structure doubled in the (x,y) plane and the corresponding BZ. The comparison between $(\mathrm{g})$ the electronic band diagram without SOC for the initial structure, and the same band diagram (h) computed using the BZ of the expanded structure, highlights the band folding from the M-point to the $\Gamma$-point (see text).

degeneracy lifting induced by lattice strain and tilts of the inorganic octahedra, these band diagrams are directly connected to the reference's one. The electronic states close to the electronic band gap are folded from $\mathrm{R}$ of the cubic phase BZ to $\Gamma$ of the orthorhombic supercell BZ. In addition, electronic states from $\mathrm{M}$ of the $P m \overline{3} m$ BZ are also folded back to Г. ${ }^{39,46}$

Incidentally, $\operatorname{Pm} \overline{3} m$ is experimentally observed at high temperature for the family of compounds $\mathrm{CH}_{3} \mathrm{NH}_{3} \mathrm{PbX}_{3}(\mathrm{X}=\mathrm{I}, \mathrm{Br})$. In that case, the positions of all the atoms of the organic cations are dynamically averaged, leading to the occupation of the same spatial place at the center of the cubic cell. As far as the electronic structure is concerned, inorganic cation, such as $\mathrm{Cs}^{+}$or $\mathrm{Na}^{+}$, can substitute the organic cation and address the disorder issue without much effect on the electronic band diagram close to the band gap. ${ }^{39}$ This allows investigating the CBM and valence band maximum (VBM) electronic states, which mainly 
involve atomic orbitals of the inorganic octahedra.

To the best of our knowledge, no highly symmetric phase for 2D HOP has ever been reported. Therefore, the procedure applied to 3D materials cannot be directly used to investigate layered structures. An ad hoc attempt to tackle this issue is to design an artificial high symmetry $D_{4 h}$ reference phase by putting $\mathrm{Cs}^{+}$cations close to the inorganic layer thus mimiking the ionic interaction (Figure 1-e). The CBM and VBM are then obtained at the M-point (Figure 1-g) in agreement with the early findings obtained by Knutson and coworkers on an idealized undistorted $\mathrm{SnI}_{4}^{2-}$ perovskite sheet. ${ }^{58}$ This can be intuited from atomic orbital hybridization appearing in both VBM and CBM and showing up at the BZ edge. ${ }^{46}$ When the M-X distance increases, the anti-bonding character of VBM decreases, as does the bonding character of the CBM, resulting in a larger band gap. By doubling the cell in the (x,y) plane (Figure 1-f), the CBM and VBM electronic states fold back

from the M- to the $\Gamma$-point (Figure 1-h). Such a cell doubling is invariably observed and reveals either a symmetry reduction or octahedral tilting in the layered HOP. The actual structure, however, is established by the temperature as well as by the chemical strain of the organic part. The simplest structures are observed for some compounds, which crystallize in a monoclinic $P 2_{1} / c$ structure (Table 1 ). Noteworthy, these structures contain a very large number of atoms per unit cell (over 100) exceeding by far those comprised within Pnma, $R 3 m, A m m 2$ or $I 4 / m c m$ structures, which are usually considered for the simulation of 3D HOP crystals. Moreover, layered HOP crystals often exhibit an additional cell doubling along the stacking axis, leading, for example, to the Pbca space group and twice more atoms in the unit cell (vide infra). Overall, these are important considerations for practical first-principles simulations of the layered HOP properties.

\section{Structural phase transitions and inorganic lattice distortions}

In a structure-property perspective, one expects a direct relationship between crystal structure and band gap. Space groups and computed band gaps of several layered HOP are 
summarized in Table 1. It demonstrates a counterintuitive increase in band gaps for (Bu$\left.\mathrm{NH}_{3}\right)_{2} \mathrm{MI}_{4}(\mathrm{M}=\mathrm{Pb}, \mathrm{Sn})$ with decreasing the temperature as a result of the reconstructive Pbca to Pbca phase transitions. Indeed, given the respective bonding and anti-bonding characters of CBM and VBM, a shrinking of the unit cell with decreasing temperature, should in turn reduce the band gap. A similar increase is predicted at the first order reconstructive tetragonal/orthorhombic phase transition of $\mathrm{CH}_{3} \mathrm{NH}_{3} \mathrm{PbI}_{3} \cdot{ }^{7,8}$

However, in the case of layered HOP, the specific relation between space groups involved in phase transitions, is obviously not the driving mechanism of the band gap variations. For instance, the band gap of decylammonium lead iodide (Decyl- $\left.\mathrm{NH}_{3}\right)_{2} \mathrm{PbI}_{4}{ }^{56}$ undergoes a very weak decrease along the low temperature $P 2_{1} / c$ phase $(1.39 \mathrm{eV})$ to the intermediate Pbca phase $(1.37 \mathrm{eV})$, whereas a much steeper decrease occurs at the Pbca to Pbca phase transition with $E_{g}=1.21 \mathrm{eV}$ for the room temperature structure (Table 1). These trends agree nicely with those observed in the experimental photoluminescence spectra. ${ }^{23}$ Similar low/high temperature band gap variations also occur from $P 2_{1} / c$ to $P b c a$, or from $P b c a$ to $P 2_{1} / c$, depending on the length of the aliphatic chain in alkyl-ammonium based 2D HOP. ${ }^{23,53,56}$ This is due to the fact that the actual space group in $2 \mathrm{D}$ HOP is essentially determined by the crystal packing, the flexibility and the thermal disorder of the organic moiety at high temperature. Thus, the resulting space group does not give direct indication on the octahedral tilting angles. In 3D HOP, the latter are known to be the origin of the band gap increase with decreasing temperature. ${ }^{46,59}$ Namely, due to sizeable octahedral deformations, the CBM and VBM are destabilized and stabilized, respectively, thus enlarging the band gap. This behavior, well documented for 3D materials, is yet to be observed in layered HOP. Therefore, additional temperature dependent structural data and optical absorption spectra on 2D HOP crystals would help to rationalize the relationships between band gap and structural features.

Meanwhile, a better insight in band gap evolutions of layered HOP can be gained by focusing on structural parameters of the inorganic part. Here, we report axial (i.e. out-of- 
plane) $d_{M-I}^{a x}$ and equatorial (i.e. in-plane) $d_{M-I}^{e q}$ distances as well as in-plane M-I-M angles for several metal-iodide systems (Table 1). For instance, the slight changes in the axial and equatorial distances at the structural phase transitions in the $\left(\mathrm{Bu}-\mathrm{NH}_{3}\right)_{2} \mathrm{MI}_{4}$ compounds with $\mathrm{M}=\mathrm{Pb}, \mathrm{Sn}$, are obviously not the critical parameters for the band gap variation. Noteworthy, all the reported in-plane M-I-M angles significantly differ from $180^{\circ}$, which is expected for an ideal $D_{4 h}$ reference phase (Figure 1-e). This had already been discussed by Knutson and coworkers based on extended Hückel tight-binding calculations and spectroscopic data in a series $\left(\mathrm{R}-\mathrm{NH}_{3}\right)_{2} \mathrm{SnI}_{4}$ of layered perovskites. ${ }^{58}$ They demonstrated that the Sn-I-Sn bond angle is the dominant structural factor that controls the variation in the band gap'.

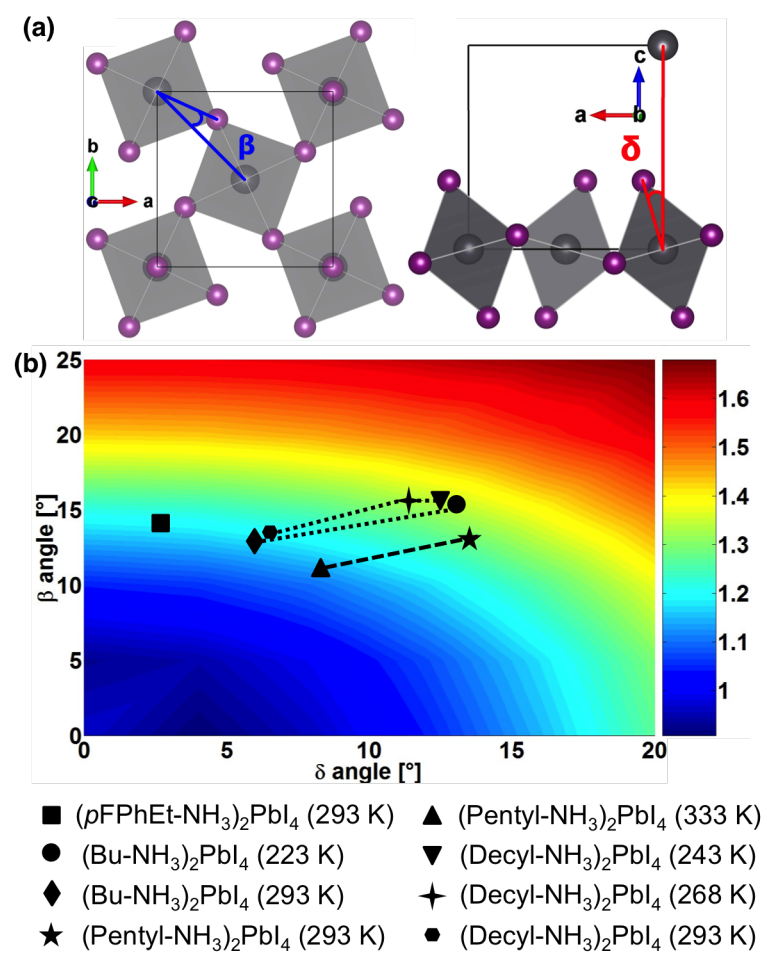

Figure 2: (a) Schematic representations of the in-plane projection of the M-M-X angle $\beta$ and the $\delta$ angle chosen to characterize the in-plane and out-of-plane octahedral tilting. (b) Color map of the computed electronic band gap including SOC for a distorted $(\mathrm{Cs})_{2} \mathrm{PbI}_{4}$ reference structure (Figure 1-e) with axial $d_{P b-I}^{a x}$ and equatorial $d_{P b-I}^{e q}$ distances fixed to $3.18 \AA$, as a function of $\beta$ and $\delta$ octahedral tilting angles. $\beta$ and $\delta$ octahedral tilting angles for several layered structures are reported.

To further rationalize such findings, in the same that it had been done for 3D HOP by 
Filip et al. ${ }^{59}$ we introduce two different angles that vanish whenever ideal $D_{4 h}$ is satisfied: (i) the in-plane projection of the M-M-X angle $\beta$ (Figure 2-a) characteristic of in-plane rotation and (ii) the $\mathrm{MX}_{6}$ octahedral tilting angle $\delta$ (Figure 2-a) characteristic of out-ofplane displacement. They are defined considering an in-plane iodine atom with fractional atomic coordinate $(1 / 4,1 / 4,0)$ that moves to position $(1 / 4-u, 1 / 4+u,-w)$. Then, the in-plane lattice parameter $a$ (here, we assume $a=b$ ), angles $\beta$ and $\delta$ satisfy the following trigonometrical relationships:

$$
\begin{array}{r}
a=\frac{d_{P b-I}}{\sqrt{(1 / 4-u)^{2}+(1 / 4+u)^{2}+(w c / a)^{2}}}, \\
\tan \beta=4 u, \\
\tan \delta=4 \frac{w c}{a},
\end{array}
$$

where $u, w$ are the fractional atomic displacements and $c$ is the lattice parameter along the stacking axis. In order to highlight the influence of $\beta$ and $\delta$ angles, we designed a simplified model starting from an undistorted $\mathrm{Cs}_{2} \mathrm{PbI}_{4}$ reference structure with fixed axial and equatorial distances set to $d_{P b-I}^{a x}=d_{P b-I}^{e q}=3.18 \AA$. Figure 2-b is a color map of the computed electronic band gap (including SOC) as a function of the $\beta$ and $\delta$ angles. The $\beta$ and $\delta$ angles for the experimental structures of $\left(p \mathrm{FPhEt}-\mathrm{NH}_{3}\right)_{2} \mathrm{PbI}_{4}$ at room temperature, ${ }^{51,52}$ $\left(\mathrm{Bu}-\mathrm{NH}_{3}\right)_{2} \mathrm{PbI}_{4}$ at low and room temperature, ${ }^{53}$ of $P 2_{1} / c$ structure of $\left(\text { Pentyl- } \mathrm{NH}_{3}\right)_{2} \mathrm{PbI}_{4}$ and its high temperature $(333 \mathrm{~K}) \mathrm{Pbca}$ structure $^{53}$ are reported on the color map, together with the low temperature $P 2_{1} / c$ structure of $\left(\text { Decyl- } \mathrm{NH}_{3}\right)_{2} \mathrm{PbI}_{4}$ as well as $\mathrm{Pbca}$ structures taken at $268 \mathrm{~K}$ and $293 \mathrm{~K} .{ }^{53}$ The corresponding band gaps (Table 1) agree nicely with the prediction of the simplified model, emphasizing the relevance of $\beta$ and $\delta$ as key parameters for band gap tuning in 2D HOP. Moreover, as evidenced in Table 1 and Figure 2, the most important band gap variations between high and low temperature phases are related to the out-of-plane tilt angle $\delta$. We stress that similar deformations of the octahedron are also observed along the $I 4 / m c m$ to Pnma phase transition in $\mathrm{CH}_{3} \mathrm{NH}_{3} \mathrm{PbX}_{3}$ accompanied by 
similar band gap variations.

\section{Loss of inversion symmetry and Rashba effect}

A possible splitting of the spinor bands due to a loss of inversion symmetry in 3D HOP pseudocubic structures was proposed early by several theoretical groups. ${ }^{60-65}$ Various Rashba, Dresselhaus or mixed Rashba-Dresselhaus scenarios have been introduced, which depend on the particular group-subgroup relationships between the parent centrosymmetric cubic $\operatorname{Pr} \overline{3} m$ phase and the non-centrosymmetric child phase. ${ }^{65}$ The spinor splittings away from the R-point of the cubic phase are predicted to be very large, because the SOC effect is giant in lead-based HOP by comparison to conventional semiconductors. However, existence of Rashba and/or Dresselhaus effect in 3D and/or 2D HOP still needs to be experimentally confirmed.

In the case of layered HOP, a similar behavior has already been predicted theoretically. $\left(\mathrm{PhMe}-\mathrm{NH}_{3}\right)_{2} \mathrm{PbCl}_{4}$ crystallizes at room temperature in the ferroelectric $C m c 2_{1}$ phase, often encountered in layered HOP. ${ }^{66,67}$ This material crystallizes in a high temperature $\mathrm{Cmca}$ space group $\left(D_{2 h}\right)$, with a disordered and centrosymmetric structure. Compared to the $D_{4 h}$ reference structure, this non-polar structure exhibits already significant distortion with an in-plane cell doubling leading to electronic band folding from the $\mathrm{M}$ - to the $\Gamma$-point (Figure 1) as well as a cell doubling along the stacking axis. The $C m c 2_{1}$ ferroelectric phase results in a further symmetry reduction to $C_{2 v}$, compatible with a Rashba-like effect. ${ }^{65}$ Hence, the $\mathrm{Cmca}$ to $\mathrm{Cmc2} 2_{1}$ transition leads to a band splitting around the $\Gamma$-point. In a classical Rashba picture, it would be observable in the $\Gamma \rightarrow \mathrm{Y}$ and $\Gamma \rightarrow \mathrm{X}$ directions. However, in contrast to classical heterostructures of semiconductors, the $C_{2}$ axis is not the stacking axis. Therefore, the splitting that should be observed along $\Gamma \rightarrow \mathrm{X}$ vanishes, while, as expected, the dispersion along $\Gamma \rightarrow \mathrm{Z}$, i.e. the high symmetry axis, carries no splitting (Figure 3-b). This specific feature leads to an unusual spin texture around $\Gamma$-point (Figure 3-c) with an inplane single direction for spins instead of the expected circulation associated with a Rashba 


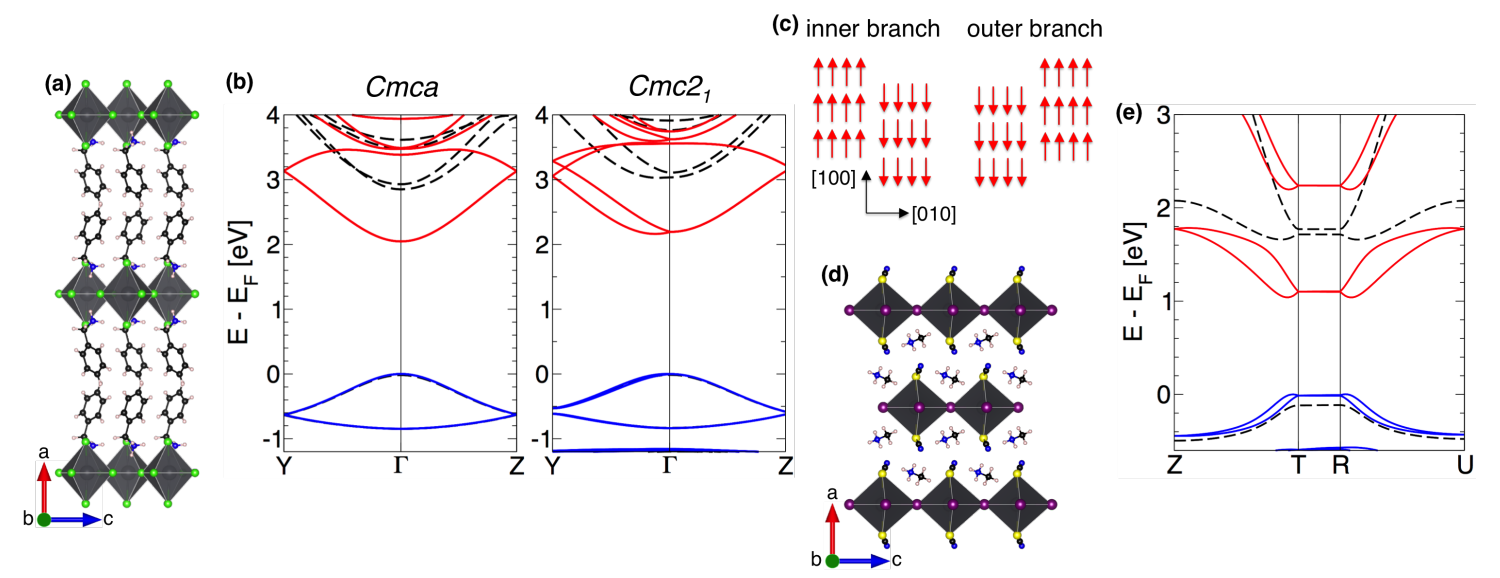

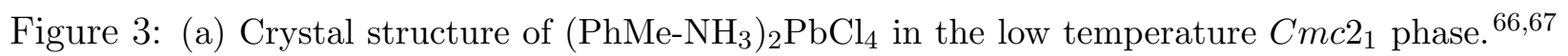
$\mathrm{Pb}, \mathrm{Cl}, \mathrm{N}, \mathrm{C}$ and $\mathrm{H}$ atoms are depicted in grey, green, blue, black and white, respectively. (b) Band structures computed without (dashed black line) and with SOC (blue and red solid lines) in the high-temperature centrosymmetric $C m c a$ phase (left) and low-temperature ferroelectric $C m c 2_{1}$ phase (right). (c) Spin textures for the inner and outer branches of the conduction band of (PhMe$\left.\mathrm{NH}_{3}\right)_{2} \mathrm{PbCl}_{4}$ in the $C m c 2_{1}$ phase. A similar behavior is computed for $\left(\mathrm{CH}_{3} \mathrm{NH}_{3}\right)_{2} \mathrm{~Pb}(\mathrm{SCN})_{2} \mathrm{I}_{2}$ in the Pmn2 $2_{1}$ group. (d) Crystal structure of $\left(\mathrm{CH}_{3} \mathrm{NH}_{3}\right)_{2} \mathrm{~Pb}(\mathrm{SCN})_{2} \mathrm{I}_{2} \cdot{ }^{68} \mathrm{~Pb}, \mathrm{I}, \mathrm{S}, \mathrm{N}, \mathrm{C}$ and $\mathrm{H}$ atoms are depicted in grey, purple, yellow, blue, black and white, respectively. (e) Band structures computed without (dashed black line) and with SOC (blue and red solid lines).

effect.

Recently, another example of layered $\mathrm{HOP},\left(\mathrm{CH}_{3} \mathrm{NH}_{3}\right)_{2} \mathrm{~Pb}(\mathrm{SCN})_{2} \mathrm{I}_{2}$ (Figure 3-d), has been synthesized ${ }^{68}$ and analyzed. ${ }^{30,69,70}$ It belongs to the non-centrosymmetric Pmn2 $2_{1}$ group, with a $S_{2}$ axis along $c$. Hence, it can present a splitting similar to the one observed with (PhMe$\left.\mathrm{NH}_{3}\right)_{2} \mathrm{PbCl}_{4}$. Figure 3-e displays the computed band structure. ${ }^{70}$ The system features a slightly indirect band gap around both $\mathrm{T}(0,1 / 2,1 / 2)$ and $\mathrm{R}(1 / 2,1 / 2,1 / 2)$. Around those points, we can observe a SOC-induced splitting characteristic of a Rashba-like effect, as confirmed by the spin texture that is identical to the one obtained with $\left(\mathrm{PhMe}-\mathrm{NH}_{3}\right)_{2} \mathrm{PbCl}_{4}$ (Figure 3-c).

In view of this 'incomplete' Rashba-like effects, one may wonder whether such effect might ever be fully observed in a 2D layered HOP at room temperature. A first experimental demonstration of a Rashba-like effect in a layered HOP structure, will perhaps be realized by applying an out-of-plane external field on a nanometer scale layer of a 3D HOP as proposed by some of us recently in a spin-valve architecture. ${ }^{65}$ 


\section{Dielectric, optical and excitonic properties}

The first success of layered HOP is related to the unique optoelectronic properties shown even at room temperature, including excellent luminescence efficiencies, white light emission ${ }^{71}$ and non-linear optical properties in microcavities. ${ }^{52,72}$ Such spectacular features are directly related to the dielectric properties of this composite material.

Following the similarity with semiconductor heterostructures, dielectric constants in $2 \mathrm{D}$ HOP can be analyzed with classical models relying on the abrupt interface approximation. ${ }^{73,74}$ However, as recently proposed by some of us, ${ }^{75-78}$ one can go one step beyond and adopt an ab initio atomistic description of the material to reach the high-frequency (including only the electron gas contributions) and the static (including both the electron gas and the polar phonons contributions) dielectric profiles. It allows description at the atomic level and without any empirical input, the dielectric confinement emerging from the contrast between inorganic and organic layers or the surface effects in colloidal nanostructures. ${ }^{78}$

As a representative example of layered HOP, the high-frequency dielectric profile of (Decyl- $\left.\mathrm{NH}_{3}\right)_{2} \mathrm{PbI}_{4}$ (Figure 4-a) is depicted in Figure 4-b. We observe clear dielectric contrast between the low dielectric constant of the organic part, ca. 2.2, and the high dielectric constant of the inorganic part, $c a$. 5.0. The value obtained for the organic layer is in excellent agreement with experimental data. ${ }^{79}$ For the inorganic part, the value is slightly lower than the bulk value of the natural 3D reference material $\mathrm{CH}_{3} \mathrm{NH}_{3} \mathrm{PbI}_{3}$, i.e. 5.5 , but higher than the one obtained for a model slab built with one layer of the same 3D reference material, i.e. $4.2{ }^{78}$ This discrepancy highlights the importance of an atomic description for dielectric profiles. By including the motion of ions in the presence of an electric field, we can also compute the static dielectric constant that describes not only the effect on the electron gas but also the contribution from polar phonons. For example, in the case of (Decyl- $\left.\mathrm{NH}_{3}\right)_{2} \mathrm{PbI}_{4}$, this does not affect the organic region. On the other hand, the inorganic region magnitude increases up to 15. Meanwhile, it remains significantly smaller compared to the one obtained for the bulk 3D material $\mathrm{CH}_{3} \mathrm{NH}_{3} \mathrm{PbI}_{3}\left(\epsilon_{\infty}=25\right)$. This distinct behavior can be ascribed to 


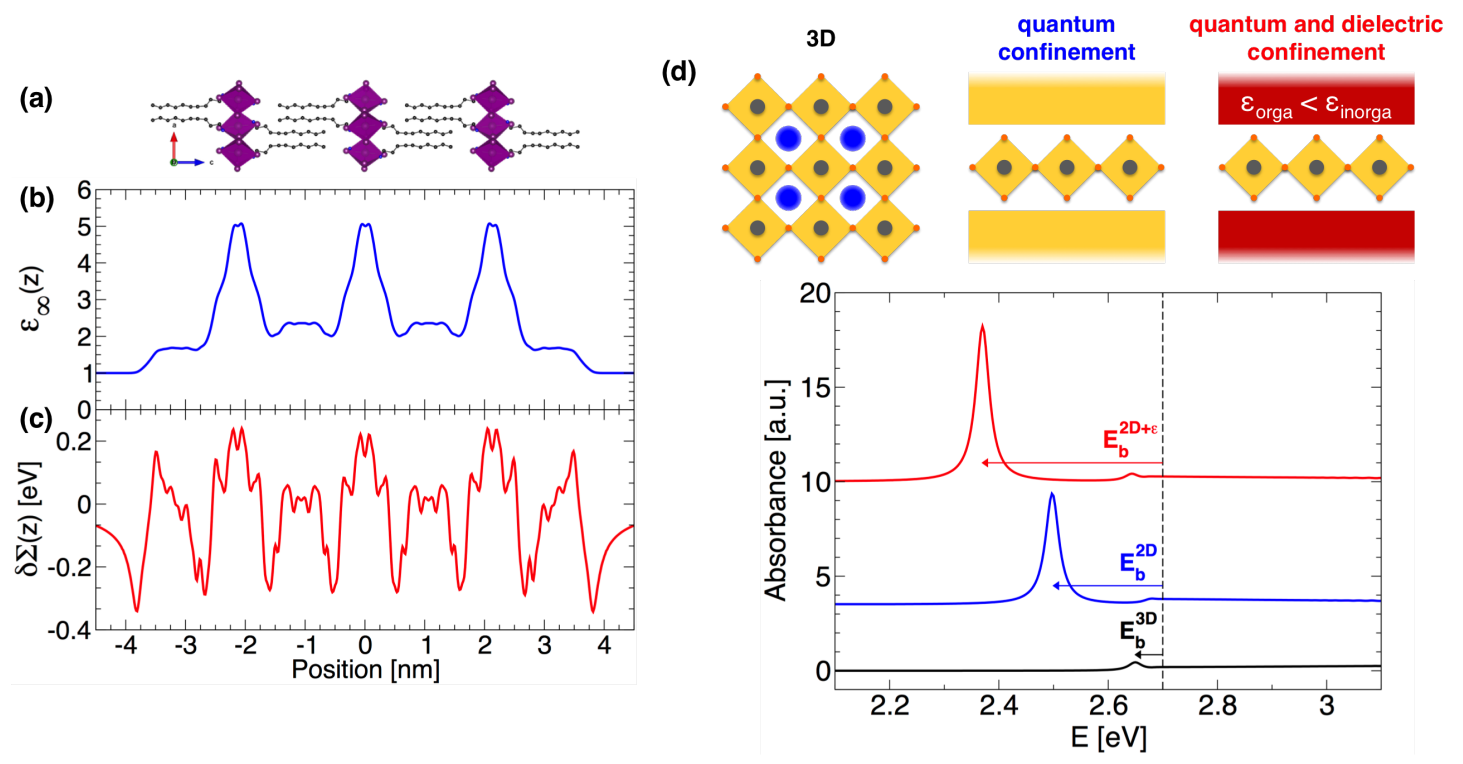

Figure 4: (a) Slab of (Decyl- $\left.\mathrm{NH}_{3}\right)_{2} \mathrm{PbI}_{4}$. $\mathrm{Pb}, \mathrm{I}, \mathrm{N}$ and $\mathrm{C}$ atoms are depicted in grey, purple, blue and black, respectively. $\mathrm{H}$ atoms are not displayed for clarity. High-frequency (b) dielectric $\epsilon_{\infty}(z)$ and (c) self-energy $\delta \Sigma^{2 D}(z)$ profiles. (d) Optical absorption computed using the k.p/BSE approach for a model HOP considered as a 3D material with $\epsilon_{\infty}=5.0$ (black line), a 2D material with only quantum confinement and $\epsilon_{\infty}=5.0$ (blue line), a 2D material with both quantum and dielectric confinement (red line). In the latter, the computed dielectric profile of (Decyl- $\left.\mathrm{NH}_{3}\right)_{2} \mathrm{PbI}_{4}$ (Figure 4-b) is used. In all cases, the band gap is set to $2.7 \mathrm{eV}$, being the value extrapolated at room temperature from available experimental data, ${ }^{23}$ and the reduced effective mass is set to 0.093 .

the difference in the phonon structures for both structures.

The optical absorption is related to the dielectric constant through the Kramers-Kronig relations. In the pseudocubic phase of 3D HOP, a purely isotropic optical activity is predicted by symmetry. On the other hand, the symmetry analysis of $\mathrm{CB}$ and VB in layered HOP shows a pure transverse electric (TE) optical activity at the band gap energy. ${ }^{27}$

A further contribution to the optical absorption close to the band gap is attributed to the presence of the excitonic electron-hole interaction. In the typical $3 \mathrm{D} \mathrm{HOP} \mathrm{CH}_{3} \mathrm{NH}_{3} \mathrm{PbI}_{3}$, the exciton binding energy at low temperature $(4 \mathrm{~K})$ lies around $50 \mathrm{meV}$. However, it has been shown that this value does not describe excitonic effects at room temperature. ${ }^{45}$ Indeed, the exciton is screened above the tetragonal to orthorhombic structural phase transition of $\mathrm{CH}_{3} \mathrm{NH}_{3} \mathrm{PbI}_{3}(\mathrm{ca} .160 \mathrm{~K}) .{ }^{7,8,48}$ The discrepancy between low and high temperature experimental binding energies stems from an additional exciton screening, related to inter- 
actions between polar phonons, which leads to an increased effective dielectric constant. The thermally activated disorder of the polar cation's orientations associated with the phase transition, further screens the excitonic interaction. The paradox between helium and room temperature measurements was finally resolved by the observation of the co-existence of Wannier-like and bound exciton species, observed in the 50-100 K temperature range. ${ }^{7}$ The helium temperature exciton line was attributed to a bound exciton with a long lifetime, possibly arising from a strong coupling with frozen orientations of the organic cations. A path to understanding of the 3D HOP excitonic structure exemplifies the need of joint spectroscopic and theoretical studies for deeper insights in optoelectronic properties of such complex materials. It is well known that the electron-hole interaction is deeply impacted by the electron gas dimensionality. This prompts for further investigations of the excitonic properties in layered HOP.

In semiconductors or insulators, the standard approach accounting for electron-hole interaction relies on the use of the BSE built on the monoelectronic states computed at the DFT level or, even better, at the DFT+GW level. ${ }^{80}$ Indeed, in many-body perturbation theory, the energy corresponding to a single-particle removal or addition, i.e. the quasiparticle energy $\epsilon^{Q P}$, is obtained from the GW self-energy operator in which the exchange-correlation effects are contained through the one-electron Green function $G$ and the screening of the Coulomb interaction $\mathrm{W}$,

$$
\Sigma\left(\vec{r}, \overrightarrow{r^{\prime}}, \epsilon^{Q P}\right)=i \int \frac{d E^{\prime}}{2 \pi} e^{-i \delta E^{\prime}} G\left(\vec{r}, \overrightarrow{r^{\prime}}, E-E^{\prime}\right) W\left(\vec{r}, \overrightarrow{r^{\prime}}, E^{\prime}\right)
$$

However, in the perturbative BSE/DFT (or DFT+GW) scheme, screening of the electronhole interaction due to atomic motion is not taken into account. Thus BSE is more relevant at low temperature where, for example, cation motions are expected to be frozen. Moreover, from a technical point of view, the $\mathrm{DFT}+\mathrm{GW}+\mathrm{BSE}$ scheme is computationally heavy. In fact, it is roughly 100 times more numerically demanding compared to an equivalent stan- 
dard DFT calculations. The DFT+GW scheme including SOC has been applied on several occasions to 3D HOP. ${ }^{60,62}$ More recently, Bokdam et al. used the same DFT+GW+BSE scheme with a refined description of the one-electron energies. ${ }^{81}$ Unfortunately, for complex materials such as layered HOP, which have a large number of atoms per unit cell as compared to classical semiconductors, current computational resources are generally not sufficient to afford full DFT + GW + BSE level of theory. Concerning the issue of the band gap, Fraccarollo et al. recently proposed an additive scheme where SOC and GW corrections are included separately. ${ }^{82}$ Here, to simulate excitonic effects in layered HOP, we suggest the use of a semi-empirical framework, such as the $\mathbf{k} \cdot \mathbf{p}$ model combined with $a b$ initio calculations.

In the literature, the enhanced exciton binding energy of layered HOP is mainly attributed to dielectric confinement effects. ${ }^{73,74}$ This is the case of a pioneering contribution by Muljarov et al., ${ }^{74}$ where the exciton binding energies were calculated using a classical resolution of the BSE. Unfortunately, this approach relied on an abrupt dielectric profile and necessary parameters were fitted to experimental data obtained on bulk 3D HOP. Moreover, to avoid unphysical divergences related to abrupt dielectric interfaces, ${ }^{76}$ an artificial transitional layer at the interface between the organic and inorganic parts had to be considered. ${ }^{74}$ By outlining a scheme allowing for the computation of a DFT-based dielectric profile, we herein propose an alternative strategy to model absorption properties, including excitonic effects, of 3D and layered HOP.

Starting from a reference 3D HOP, the 2D band gap can be estimated from theoretical calculations by using the following decomposition:

$$
E_{g} \approx E_{g, D F T}^{3 D}+\Sigma^{3 D}+\delta E_{g, D F T}^{2 D}+\delta \Sigma^{2 D}
$$

where $E_{g, D F T}^{3 D}+\Sigma^{3 D}$ is the $3 \mathrm{D}$ band gap, including the self-energy correction. $\delta E_{g, D F T}^{2 D}$ accounts for the effects of quantum confinement and lattice distortions occurring in the $2 \mathrm{D}$ material. Thus, $E_{g, D F T}^{3 D}+\Sigma^{3 D}+\delta E_{g, D F T}^{2 D}$ is the $2 \mathrm{D}$ band gap in principle available 
from DFT calculations. However, band gaps are usually not well reproduced by plain DFT calculations. If more accurate strategies such as GW corrections are out of reach, one might use an experimentally determined gap or rely on the use of hybrid functionals instead. Last, $\delta \Sigma^{2 D}$ contains additional corrections related to dielectric confinement.

Self-energy corrections can be computed for monoelectronic states near the band gap starting from the dielectric profile:

$$
\delta \Sigma\left(z_{0}\right)=\frac{e_{0}}{4 \pi} \int_{0}^{\infty}\left(V\left(q, z, z_{0}\right)-V_{b u l k}\left(q, z, z_{0}\right)\right)_{z \rightarrow z_{0}} q \mathrm{~d} q .
$$

where $\mathrm{e}_{0}$ is a test charge at position $\mathrm{z}_{0}, V$ and $V_{\text {bulk }}$ the electrostatic potential in the heterostructure and in the bulk, respectively. Back to $\mathrm{GW}, \delta \Sigma\left(z_{0}\right)$ is equivalent to the one particle potential profile acting on the test charge. $V$ is then obtained by solving the inhomogeneous Poisson equation including the dielectric constant profile $\epsilon(z)$ :

$$
\frac{\partial}{\partial z}\left(\epsilon_{\infty}(z) \frac{\partial}{\partial z}\left(V\left(q, z, z_{0}\right)\right)\right)-q^{2} \epsilon_{\infty}(z) V\left(q, z, z_{0}\right)=-e_{0} \delta\left(z-z_{0}\right)
$$

A detailed description of the procedure is to be found in references 76 and 78 . The example of (Decyl- $\left.\mathrm{NH}_{3}\right)_{2} \mathrm{PbI}_{4}$, Figure 4-c displays the self-energy profile $\delta \Sigma(z)$ computed according to the dielectric profile $\epsilon_{\infty}(z)$. At the position of the inorganic sheet, the self-energy correction is about $+0.2 \mathrm{eV}$ and 10 times less $c a .+0.02 \mathrm{eV}$ for the organic part. The semi-classical evaluation of $\delta \Sigma^{2 D}$, both for the $\mathrm{CB}$ and the VB states, can be obtained by computing the following integrals

$$
\delta \Sigma_{C B(V B)}^{2 D}=\int \delta \Sigma^{2 D}(z) \rho_{C B(V B)}(z) d z
$$

Here $\rho_{C B(V B)}(z)$ is the electronic density profile for the CB (VB) state. The integral is computed over a unit cell, taking the central part of $\delta \Sigma^{2 D}(z)$. We then obtain the total band gap correction $\delta \Sigma^{2 D}=\delta \Sigma_{C B}^{2 D}+\delta \Sigma_{V B}^{2 D}$ and deduce the band gap $E_{g}$ from Eq. (2). 
Finally, to compute the full absorption spectrum including excitonic effects, we still need to include the electron-hole interaction. Here we provide a brief description of the approach that will be described at more length elsewhere. In short, it can be implemented semi-empirically by solving the BSE based on the work by Chuang et al. ${ }^{83}$ considering $z=z_{0}=0$ due to the $2 \mathrm{D}$ character of the material of interest. The Green's function of the exciton is solved according to equation (19) of reference 83. The optical susceptibility is computed using equation (14) of the same publication. The effective mass approximation is used for the energies (equation (11) of reference 83). Those can be evaluated from the computed band structure. Corresponding wavefunctions, given in (8a) and (8b) of reference 83), are also deduced from the DFT computed electron densities at the center of the BZ. This scheme, in principle, calculates the electron-hole Coulombic interaction without any experimental input. In the case of typical layered HOP, evaluation of GW corrections is out of reach due to large system size. Subsequently, we can rely on the experimental data to appraise these quantities. Using again (Decyl- $\left.\mathrm{NH}_{3}\right)_{2} \mathrm{PbI}_{4}$ as an example, we extrapolate the experimental band gap of $2.88 \mathrm{eV}$ recorded at $1.6 \mathrm{~K}$ to $2.7 \mathrm{eV}$ at room temperature from reference 23 .

In order to demonstrate the dimensionality and dielectric effects, we use different ingredients for a 3D perovskite structure (Figure 4-d, black line). The optical absorption is enhanced at the exciton resonance and an exciton binding energy of $50 \mathrm{meV}$ is computed, when choosing a reduced effective mass of 0.093, a value consistent with magneto-absorption measurements. ${ }^{8}$ In a second step, we restore the $2 \mathrm{D}$ character of $\left(\text { Decyl- } \mathrm{NH}_{3}\right)_{2} \mathrm{PbI}_{4}$ but do not take into account the dielectric properties as computed in Figure 4-c. Here, the specific effect of quantum confinement on the exciton is evidenced as shown in Figure 4-d (blue line). As expected when going from a 3D to $2 \mathrm{D}$ structure, the exciton binding energy increases from 50 to $200 \mathrm{meV}$. When further including the dielectric confinement contribution, a large exciton binding energy of $330 \mathrm{meV}$ is predicted (Figure 4-d, red line), in fair agreement with available experimental data. ${ }^{23}$ These three representative calculations illustrate the relative 
impacts of quantum and dielectric confinements. We emphasize that an accurate description of excitonic properties cannot avoid the precise delineation of the dielectric contrast.

\section{Conclusion}

3D HOP are current photovolatic superstars. Superb performances have motivated intense investigations and shed light on their structural, electronic and optical properties. Meanwhile, 2D HOP, that used to be the most popular members of the family, have been in the shadow of this sudden success. However, they are coming back thanks to better photo- and environmental stability in solar cells and attractive light-emission properties. Furthermore, compared to their 3D counterparts, they represent a greater playground for chemists to investigate.

In this review, we outline a systematic comparison of the structural, electronic and optical properties of the 3D HOP, and their 2D layered analogs. In both cases, SOC has a dramatic impact on the conduction bands, while it typically modifies the valence band in conventional semiconductors. A specific feature of layered materials is their quantum-well-like behavior that approaches a type-I quantum confinement, however, without the superlattice effects. These composite structures are also characterized by strongly anisotropic electronic, optical and charge carrier transport properties. Similarly to the reference pseudocubic $\operatorname{P} m \overline{3} m$ phase already established for 3D HOP, here we introduce the hypothetical $D_{4 h}$ structure, which can be used to rationalize the properties of layered HOP. For instance, a comparable Brillouin zone folding can be considered to facilitate analysis of structure/property relationships. Finally, we survey dielectric and excitonic properties of layered 2D. To that end, we propose an original theoretical treatment based on an $a b$ initio description to supersede the usual $\mathrm{DFT}+\mathrm{GW}+\mathrm{BSE}$ treatment that cannot be applied to layered HOP due to currently intractable computational cost. The former also allows for a detailed description at the atomistic level, the interface and surface effects, however, at a greatly reduced computational cost. 
The HOP family still has an enormous potential for various optoelectronic applications, yet further progress requires intense investigations. Over the last two years, our understanding of 3D HOP properties has made a breakthrough thanks to the possibility of growing large monocrystals. The similar crystal engineering advance of layered HOP would also greatly benefit the field. In particular, it would allow for analysis of structures and phase transitions by enabling many spectroscopic studies (IR, Raman, Brillouin, neutron scattering, etc.). It would also confirm or overturn the presence of Rashba effect that, to this moment, has not been observed in either layered or 3D HOP. 3D structures are very constraining regarding the size of the cation, leading to severe restrictions over the choice of the organic moiety. The situation is different with 2D HOP, where chemical engineering can follow many paths that have yet to be discovered. The wealth of HOP is not restricted to 3D and 2D systems. In this tremendous playground, many dimensions are present. For instance, among those very promising features are delivered by $3 \mathrm{D} / 2 \mathrm{D}$ structures, where the inorganic sheet counts more than one layer of octahedra.

\section{Acknowledgement}

The work at FOTON is supported by Agence Nationale pour la Recherche (SNAP and SuperSansPlomb projects) and was performed using HPC resources from GENCI-CINES/IDRIS Grant 2016-c2012096724. The work at ISCR is supported by Agence Nationale pour la Recherche (TRANSHYPERO project). J.E.s work is supported by the Fondation dentreprises banque Populaire de lOuest under Grant PEROPHOT 2015. The work at Los Alamos National Laboratory (LANL) was supported by LANL LDRD program and was partially performed at the Center for Nonlinear Studies and at the Center for Integrated Nanotechnologies, a U.S. Department of Energy, Office of Science user facility. The Groningen team would like to acknowledge funding from European Research Council (ERC Starting Grant Hy-SPOD No. 306983) and by the Foundation for Fundamental Research on Matter (FOM), which is part of The Netherlands Organization for Scientific Research (NWO), un- 
der the framework of the FOM Focus Group Next Generation Organic Photovoltaics. ICN2 acknowledges support from the Severo Ochoa Program (MINECO, Grant SEV-2013-0295). This project received funding from the European Union's Horizon 2020 research and innovation programme under the grant agreement no. 687008. The information and views set out in this publication are those of the authors and do not necessarily reflect the official opinion of the European Union. Neither the European Union institutions and bodies nor any person acting on their behalf may be held responsible for the use which may be made of the information contained therein. 


\section{References}

1. Kojima, A.; Teshima, K.; Shirai, T.; Miyasaka, T. Organometal Halide Perovskites as Visible-Light Sensitizers for Photovoltaic Cells. J. Am. Chem. Soc. 2009, 131, 6050.

2. Lee, M. M.; Teuscher, J.; Miyasaka, T.; Murakami, T. N.; Snaith, H. J. Efficient Hybrid Solar Cells Based on Meso-Superstructured Organometal Halide Perovskites. Science 2012, 338, 643-647.

3. Kim, H.-S.; Lee, C.-R.; Im, J.-H.; Lee, K.-B.; Moehl, T.; Marchioro, A.; Moon, S.-J.; Humphry-Baker, R.; Yum, J.-H.; Moser, J. E. et al. Lead Iodide Perovskite Sensitized All-Solid-State Submicron Thin Film Mesoscopic Solar Cell with Efficiency Exceeding 9\%. Sci. Rep. 2012, 2, 591.

4. Burschka, J.; Pellet, N.; Moon, S.-J.; Humphry-Baker, R.; Gao, P.; Nazeeruddin, M. K.; Grätzel, M. Sequential deposition as a route to high-performance perovskite-sensitized solar cells. Nature 2013, 499 , $316-319$.

5. Liu, M.; Johnston, M. B.; Snaith, H. J. Efficient Planar Heterojunction Perovskite Solar Cells by Vapour Deposition. Nature 2013, 501, 395-398.

6. Nie, W.; Tsai, H.; Asadpour, R.; Blancon, J.-C.; Neukirch, A. J.; Gupta, G.; Crochet, J. J.; Chhowalla, M.; Tretiak, S.; Alam, M. A. et al. High-efficiency solution-processed perovskite solar cells with millimeter-scale grains. Science 2015, 347, 522-525.

7. Fang, H.-H.; Raissa, R.; Abdu-Aguye, M.; Adjokatse, S.; Blake, G. R.; Even, J.; Loi, M. A. Photophysics of Organic-Inorganic Hybrid Lead Iodide Perovskite Single Crystals. Adv. Funct. Mater. 2015, 25, 23782385 .

8. Miyata, A.; Mitioglu, A.; Plochocka, P.; Portugall, O.; Wang, J. T.-W.; Stranks, S. D.; Snaith, H. J.; Nicholas, R. J. Direct measurement of the exciton binding energy and effective masses for charge carriers in organicinorganic tri-halide perovskites. Nature Phys. 2015, 11, 582-588.

9. Bi, D.; Tress, W.; Dar, M. I.; Gao, P.; Luo, J.; Renevier, C.; Schenk, K.; Abate, A.; Giordano, F.; Correa Baena, J.-P. et al. Efficient luminescent solar cells based on tailored mixed-cation perovskites. Sci. Adv. 2016, 2, e1501170.

10. Saliba, M.; Matsui, T.; Seo, J.-Y.; Domanski, K.; Correa-Baena, J.-P.; Nazeeruddin, M. K.; Zakeeruddin, S. M.; Tress, W.; Abate, A.; Hagfeldt, A. et al. Cesium-containing triple cation perovskite solar cells: improved stability, reproducibility and high efficiency. Energy Environ. Sci. 2016, doi: $10.1039 / \mathrm{c} 5 \mathrm{ee} 03874 \mathrm{j}$. 
11. http://www.nrel.gov/ncpv/images/efficiency_chart.jpg.

12. Chen, W.; Wu, Y.; Yue, Y.; Liu, J.; Zhang, W.; Yang, X.; Chen, H.; Bi, E.; Ashraful, I.; Grätzel, M. et al. Efficient and stable large-area perovskite solar cells with inorganic charge extraction layers. Science 2015, 350, 944-948.

13. Li, X.; Bi, D.; Yi, C.; Décoppet, J.-D.; Luo, J.; Zakeeruddin, S. M.; Hagfeldt, A.; Grätzel, M. A vacuum flash-assisted solution process for high-efficiency large-area perovskite solar cells. Science 2016, doi: 10.1126/science.aaf8060.

14. Razza, S.; Di Giacomo, F.; Matteocci, F.; Cina, L.; Lorenzo Palma, A.; Casaluci, S.; Cameron, P.; D'Epifanio, A.; Licoccia, S.; Reale, A. et al. Perovskite solar cells and large area modules $\left(100 \mathrm{~cm}^{2}\right)$ based on an air flow-assisted $\mathrm{PbI}_{2}$ blade coating deposition process. J. Power Sources 2015, 277, 286291.

15. Babayigit, A.; Ethirajan, A.; Muller, M.; Conings, B. Toxicity of organometal halide perovskite solar cells. Nature Mater. 2016, 15, 247.

16. Chen, P.-Y.; Qi, J.; Klug, M. T.; Dang, X.; Hammond, P. T.; Belcher, A. M. Environmentally responsible fabrication of efficient perovskite solar cells from recycled car batteries. Energy Environ. Sci. 2014, 7, 3659 .

17. Chen, P.-Y.; Qi, J.; Klug, M. T.; Dang, X.; Hammond, P. T.; Belcher, A. M. Response to the comments on "Environmentally responsible fabrication of efficient perovskite solar cells from recycled car batteries" by Po-Yen Chen, Jifa Qi, Matthew T. Klug, Xiangnan Dang, Paula T. Hammond, and Angela M. Belcher published in Energy Environ. Sci. in 2014. Energy Environ. Sci. 2015, 8, 1618.

18. Huang, W.; Manser, J. S.; Kamat, P. V.; Ptasinka, S. Evolution of Chemical Composition, Morphology, and Photovoltaic Efficiency of $\mathrm{CH}_{3} \mathrm{NH}_{3} \mathrm{PbI}_{3}$ Perovskite under Ambient Conditions. Chem. Mater. 2016, 28, 303-311.

19. Nie, W.; Blancon, J.-C.; Neukirch, A. J.; Appavoo, K.; Tsai, H.; Chhowalla, M.; Alam, M. A.; Sfeir, M. Y.; Katan, C.; Even, J. et al. Light-activated photocurrent degradation and self-healing in perovskite solar cells. Nat. Commun. 2016, 7, 11574.

20. Mitzi, D. B.; Wang, S.; Feild, C. A.; Chess, C. A.; Guloy, A. M. Conducting Layered Organic-inorganic Halides Containing ¡110¿-Oriented Perovskite Sheets. Science 1995, 267, 1473-1476. 
21. Kagan, C. R.; Mitzi, D. B.; Dimitrakopoulos, C. D. Organic-Inorganic Hybrid Materials as Semiconducting Channels in Thin-Film Field-Effect Transistors. Science 1999, 286, 945.

22. Mitzi, D. B. Synthesis, Structure, and Properties of Organic-Inorganic Perovskites and Related Materials. Prog. Inorg. Chem. 2007, 48, 1-121.

23. Ishihara, T.; Takahashi, J.; Goto, T. Optical properties due to electronic transitions in two-dimensional semiconductors $\left(\mathrm{C}_{n} \mathrm{H}_{2 n+1} \mathrm{NH}_{3}\right)_{2} \mathrm{PbI}_{4}$. Phys. Rev. B 1990, 42, 11099-11107.

24. Goto, T.; Makino, H.; Yao, T.; Chia, C. H.; Makino, T.; Segawa, Y.; Mousdis, G. A.; Papavassiliou, G. C. Localization of triplet excitons and biexcitons in the two-dimensional semiconductor $\left(\mathrm{CH}_{3} \mathrm{C}_{6} \mathrm{H}_{4} \mathrm{CH}_{2} \mathrm{NH}_{3}\right)_{2} \mathrm{PbBr}_{4}$. Phys. Rev. B 2006, 73, 115206.

25. Mitzi, D. B.; Dimitrakopoulos, C. D.; Kosbar, L. L. Structurally Tailored Organic-Inorganic Perovskites: Optical Properties and Solution-Processed Channel Materials for Thin-Film Transistors. Chem. Mater. 2001, 13, 3728-3740.

26. Sourisseau, S.; Louvain, N.; Bi, W.; Mercier, N.; Rondeau, D.; Boucher, J.-Y., F. Buzaré; Legein, C. Reduced Band Gap Hybrid Perovskites Resulting from Combined Hydrogen and Halogen Bonding at the Organic-Inorganic Interface. Chem. Mater. 2007, 19, 600-607.

27. Even, J.; Pedesseau, L.; Dupertuis, M.-A.; Jancu, J.-M.; Katan, C. Electronic model for self-assembled hybrid organic/perovskite semiconductors: Reverse band edge electronic states ordering and spin-orbit coupling. Phys. Rev. B 2012, 86, 205301.

28. Even, J.; Pedesseau, L.; Katan, C. Understanding Quantum Confinement of Charge Carriers in Layered 2D Hybrid Perovskites. ChemPhysChem 2014, 15, 3733.

29. Pedesseau, L.; Jancu, J.-M.; Rolland, A.; Deleporte, E.; Katan, C.; Even, J. Electronic properties of 2D and 3D hybrid organic/inorganic perovskites for optoelectronic and photovoltaic applications. Opt. Quant. Electron. 2014, 46, 1225.

30. Saparov, B.; Mitzi, D. B. Organic-Inorganic Perovskites: Structural Versatility for Functional Materials Design. Chem. Rev. 2016, 116, 4558-4596.

31. Smith, I. C.; Hoke, E. T.; Solis-Ibarra, D.; McGehee, M. D.; Karunadasa, H. I. A Layered Hybrid Perovskite Solar-Cell Absorber with Enhanced Moisture Stability. Angew. Chem. Int. Ed. 2014, 53, $11232-11235$. 
32. Cao, D. H.; Stoumpos, C. C.; Farha, O. K.; Hupp, J. T.; Kanatzidis, M. G. 2D Homologous Perovskites as Light-Absorbing Materials for Solar Cell Applications. J. Am. Chem. Soc. 2015, 137, 7843-7850.

33. Dou, L.; Wong, A. B.; Yu, Y.; Lai, M.; Kornienko, N.; Eaton, S. W.; Fu, A.; Bischak, C. G.; Ma, J.; Ding, T. et al. Atomically thin two-dimensional organic-inorganic hybrid perovskites. Science 2015, 349, $1518-1521$.

34. Yaffe, O.; Chernikov, A.; Norman, Z. M.; Zhong, Y.; Velauthapillai, A.; van der Zande, A.; Owen, J. S.; Heinz, T. F. Excitons in ultrathin organic-inorganic perovskite crystals. Phys. Rev. B 2015, 92, 045414.

35. Tyagi, P.; Arveson, S. M.; Tisdale, W. A. Colloidal Organohalide Perovskite Nanoplatelets Exhibiting Quantum Confinement. J. Phys. Chem. Lett. 2015, 6, 1911.

36. Protesescu, L.; Yakunin, S.; Bodnarchuk, M. I.; Krieg, F.; Caputo, R.; Hendon, C. H.; Yang, R. X.; Walsh, A.; Kovalenko, M. V. Nanocrystals of Cesium Lead Halide Perovskites (CsPbX3, X $=\mathrm{Cl}, \mathrm{Br}$, and I): Novel Optoelectronic Materials Showing Bright Emission with Wide Color Gamut. Nano Lett. 2015, 15, 3692-3696.

37. Stoumpos, C. C.; Cao, D. H.; Clark, D. J.; Young, J.; Rondinelli, J. M.; Jang, J. I.; Hupp, J. T.; Kanatzidis, M. G. Ruddlesden-Popper Hybrid Lead Iodide Perovskite 2D Homologous Semiconductors. Chem. Mater. 2016, 28, 2852.

38. Tsai, H.; Nie, W.; Blancon, J.-C.; Stoumpos, C. C.; Asadpour, R.; Harutyunyan, B.; Verduzco, R.; Crochet, J. J.; Tretiak, S.; Pedesseau, L. et al. Layered Ruddlesden-Popper perovskite solar cells with $11.2 \%$ efficiency, superior crystallinity and environmental stability. Nature doi:10.1038/nature18306.

39. Even, J.; Pedesseau, L.; Jancu, J.-M.; Katan, C. Importance of Spin-Orbit Coupling in Hybrid Organic/Inorganic Perovskites for Photovoltaic Applications. J. Phys. Chem. Lett. 2013, 4, 2999.

40. Mosconi, E.; Amat, A.; Nazeeruddin, M. K.; Grätzel, M.; De Angelis, F. First-Principles Modeling of Mixed Halide Organometal Perovskites for Photovoltaic Applications. J. Phys. Chem. C 2013, 117, 13902-13913.

41. Brivio, F.; Walker, A. B.; Walsh, A. Structural and electronic properties of hybrid perovskites for highefficiency thin-film photovoltaics from first-principles. APL Mater. 2013, 1, 042111.

42. Giorgi, G.; Fujisawa, J.-I.; Segawa, H.; Yamashita, K. Small Photocarrier Effective Masses Featuring Ambipolar Transport in Methylammonium Lead Iodide Perovskite: A Density Functional Analysis. J. Phys. Chem. Lett. 2013, 4, 4213-4216. 
43. Hohenberg, P.; Kohn, W. Inhomogeneus Electron Gas. Phys. Rev. B 1964, 136, 864-871.

44. Kohn, W.; Sham, L. J. Self-Consistent Equations Including Exchange and Correlation Effects. Phys. Rev. A 1965, 140, 1133-1138.

45. Even, J.; Pedesseau, L.; Katan, C. Analysis of Multivalley and Multibandgap Absorption and Enhancement of Free Carriers Related to Exciton Screening in Hybrid Perovskites. J. Phys. Chem. C 2014, 118, 11566.

46. Katan, C.; Pedesseau, L.; Kepenekian, M.; Rolland, A.; Even, J. Interplay of spin-orbit coupling and lattice distortion in metal substituted 3D tri-chloride hybrid perovskites. J. Mater. Chem. A 2015, 3, 9232 .

47. Even, J.; Pedesseau, L.; Katan, C.; Kepenekian, M.; Lauret, J.-S.; Sapori, D.; Deleporte, E. Solid-State Physics Perspective on Hybrid Perovskite Semiconductors. J. Phys. Chem. C 2015, 119, 10161.

48. Even, J. Pedestrian Guide to Symmetry Properties of the Reference Cubic Structure of 3D All-Inorganic and Hybrid Perovskites. J. Phys. Chem. Lett. 2015, 6, 2238.

49. Even, J.; Carignano, M.; Katan, C. Molecular disorder and translation/rotation coupling in the plastic crystal phase of hybrid perovskites. Nanoscale 2016, 8, 6222-6236.

50. Salpeter, E. E.; Bethe, H. A. A Relativistic Equation for Bound-State Problems. Phys. Rev. 1951, 84, 1232-1242.

51. Kikuchi, K.; Takeoka, Y.; Rikukawa, M.; Sanui, K. Structure and optical properties of lead iodide based two-dimensional perovskite compounds containing fluorophenethylamines. Curr. Appl. Phys. 2004, 4, 599-602.

52. Wei, Y.; Lauret, J.-S.; Galmiche, L.; Audebert, P.; Deleporte, E. Strong exciton-photon coupling in microcavities containing new fluorophenethylamine based perovskite compounds. Opt. Express 2012, 20, 10399-10405.

53. Billing, D. G.; Lemmerer, A. Synthesis, characterization and phase transitions in the inorganic-organic layered perovskite-type hybrids $\left[\left(\mathrm{C}_{n} \mathrm{H}_{2 n+1} \mathrm{NH}_{3}\right)_{2} \mathrm{PbI}_{4}\right], n=4,5$ and 6. Acta Crystallogr. B 2007, 63, 735-747.

54. Takahashi, Y.; Obara, R.; Nakagawa, K.; Nakano, M.; Tokita, J.-y.; Inabe, T. Tunable Charge Transport in Soluble Organic-Inorganic Hybrid Semiconductors. Chem. Mater. 2007, 19, 6312-6316. 
55. Mitzi, D. B. Synthesis, Crystal Structure, and Optical and Thermal Properties of $\left(\mathrm{C}_{4} \mathrm{H}_{9} \mathrm{NH}_{3}\right)_{2} \mathrm{MI}_{4}(\mathrm{M}$ $=\mathrm{Ge}, \mathrm{Sn}, \mathrm{Pb})$. Chem. Mater. 1996, 8, 791-800.

56. Lemmerer, A.; Billing, D. G. Synthesis, characterization and phase transitions of the inorganic-organic layered perovskite-type hybrids $\left[\left(\mathrm{C}_{n} \mathrm{H}_{2 n+1} \mathrm{NH}_{3}\right)_{2} \mathrm{PbI}_{4}\right], n=7,8,9$ and 10. Dalton Trans. 2012, 41, $1146-1157$.

57. Kamminga, M. E.; Fang, H.-H.; Filip, M. R.; Giustino, F.; Baas, J.; Blake, G. R.; Loi, M. A.; Palstra, T. T. M. Confinement Effects in Low-Dimensional Lead Iodide Perovskite Hybrids. Chem. Mater. 2016, 28, 4554-4562.

58. Knutson, J. L.; Martin, J. D.; Mitzi, D. B. Tuning the Band Gap in Hybrid Tin Iodide Perovskite Semiconductors Using Structural Templating. Inorg. Chem. 2005, 44, 4699-4705.

59. Filip, M. R.; Eperon, G. E.; Snaith, H. J.; Giustino, F. Steric engineering of metal-halide perovskites with tunable optical band gaps. Nat. Commun. 2014, 5, 5757.

60. Even, J.; Pedesseau, L.; Jancu, J.-M.; Katan, C. DFT and k.p modelling of the phase transitions of lead and tin halide perovskites for photovoltaic cells. Phys. Status Solidi RRL 2014, 8, 31.

61. Kim, M.; Im, J.; Freeman, A. J.; Ihm, J.; Jin, H. Switchable $\mathrm{S}=1 / 2$ and J $=1 / 2$ Rashba bands in Ferroelectric Halide Perovskites. Proc. Natl. Acad. Sci. U.S.A. 2014, 111, 6900-6904.

62. Brivio, F.; Butler, K. T.; Walsh, A.; van Schilfgaarde, M. Relativistic Quasiparticle Self-Consistent Electronic Structure of Hybrid Halide Perovskite Photovoltaic Absorbers. Phys. Rev. B 2014, 89, 155204.

63. Amat, A.; Mosconi, E.; Ronca, E.; Quarti, C.; Umari, P.; Nazeeruddin, M. K.; Grätzel, M.; De Angelis, F. Cation-Induced Band-Gap Tuning in Organohalide Perovskites: Interplay of Spin-Orbit Coupling and Octahedra Tilting. Nano Lett. 2014, 14, 3608-3616.

64. Stroppa, A.; Di Sante, D.; Barone, P.; Bokdam, M.; Kresse, G.; Franchini, C.; Whangbo, M.-H.; Picozzi, S. Tunable Ferroelectric Polarization and its Interplay with Spin-Orbit Coupling in Tin Iodide Perovskites. Nature Commun. 2014, 5, 5900.

65. Kepenekian, M.; Robles, R.; Katan, C.; Sapori, D.; Pedesseau, L.; Even, J. Rashba and Dresselhaus Effects in Hybrid Organic-Inorganic Perovskites: From Basics to Devices. ACS Nano 2015, 12, 1155711567. 
66. Braun, M.; Frey, W. Crystal Structure of Bis(benzylammonium) Lead Tetrachloride, $\left(\mathrm{C}_{7} \mathrm{H}_{7} \mathrm{NH}_{3}\right)_{2} \mathrm{PbCl}_{4}$. Z. Kristallogr. New Cryst. Struct. 1999, 214, 331-332.

67. Liao, W.-Q.; Zhang, Y.; Hu, C.-L.; Mao, J.-G.; Ye, H.-Y.; Li, P.-F.; Huang, S. D.; Xiong, R.-G. A Lead-Halide Perovskite Molecular Ferroelectric Semiconductor. Nat. Commun. 2015, 6, 7338.

68. Daub, M.; Hillebrecht, H. Synthesis, Single-Crystal Structure and Characterization of $\left(\mathrm{CH}_{3} \mathrm{NH}_{3}\right)_{2} \mathrm{~Pb}(\mathrm{SCN})_{2} \mathrm{I}_{2}$. Angew. Chem. Int. Ed. 2015, 54, 11016-11017.

69. Ganose, A. M.; Savory, C. N.; Scanlon, D. O. $\left(\mathrm{CH}_{3} \mathrm{NH}_{3}\right)_{2} \mathrm{~Pb}(\mathrm{SCN})_{2} \mathrm{I}_{2}$ : A More Stable Structural Motif for Hybrid Halide Photovoltaics? J. Phys. Chem. Lett. 2015, 6, 4594-4598.

70. Xiao, Z.; Meng, W.; Saparov, B.; Duan, H.-S.; Wang, C.; Feng, C.; Liao, W.; Ke, W.; Zhao, D.; Wang, J. et al. Photovoltaic Properties of Two-Dimensional $\left(\mathrm{CH}_{3} \mathrm{NH}_{3}\right)_{2} \mathrm{~Pb}(\mathrm{SCN})_{2} \mathrm{I}_{2}$ Perovskite: A Combined Experimental and Density Functional Theory Study. J. Phys. Chem. Lett. 2016, 7, 1213-1218.

71. Dohner, E. R.; Jaffe, A.; Bradshaw, L. R.; Karunadasa, H. I. Intrinsic White-Light Emission from Layered Hybrid Perovskites. J. Am. Chem. Soc. 2014, 136, 13154-13157.

72. Lanty, G.; Bréhier, A.; Parashkov, R.; Lauret, J.-S.; Deleporte, E. Strong exciton-photon coupling at room temperature in microcavities containing two-dimensional layered perovskite compounds. New J. Phys. 2008, 10, 065007.

73. Kumagai, M.; Takagahara, T. Excitonic and Nonlinear-optical Properties of Dielectric Quantum-well Structures. Phys. Rev. B 1989, 40, 12359.

74. Muljarov, E. A.; Tikhodeev, S. G.; Gippius, N. A. Excitons in self-organized semiconductor/insulator superlattices: PbI-based perovskite compounds. Phys. Rev. B 1995, 51, 14370.

75. Shi, N.; Ramprasad, R. Dielectric properties of ultrathin $\mathrm{SiO}_{2}$ slabs. Appl. Phys. Lett. 2005, 87, 262102.

76. Even, J.; Pedesseau, L.; Kepenekian, M. Electronic surface states and dielectric self-energy profiles in colloidal nanoscale platelets of CdSe. Phys. Chem. Chem. Phys. 2014, 16, 25182.

77. Benchamekh, R.; Gippius, N. A.; Even, J.; Nestoklon, M. O.; Jancu, J.-M.; Ithurria, S.; Dubertret, B.; Efros, A. L.; Voisin, P. Tight-binding calculations of image-charge effects in colloidal nanoscale platelets of CdSe. Phys. Rev. B 2014, 89, 035307. 
78. Sapori, D.; Kepenekian, M.; Pedesseau, L.; Katan, C.; Even, J. Quantum confinement and dielectric profiles of colloidal nanoplatelets of halide inorganic and hybrid organic-inorganic perovskites. Nanoscale 2016, 8, 6369 .

79. Richards, T. W.; Shipley, J. W. The dielectric constants of typical aliphatic and aromatic hydrocarbons, cyclohexane, cyclohexanone, and cyclohexanol. J. Am. Chem. Soc. 1919, 41, 2002-2012.

80. Hedin, L. New Method for Calculating One-Particle Greens Function with Application to Electron-Gas Problem. Phys. Rev. 1965, 139, A796.

81. Bokdam, M.; Sander, T.; Stroppa, A.; Picozzi, S.; Sarma, D. D.; Franchini, C.; Kresse, G. Role of Polar Phonons in the Photo Excited State of Metal Halide Perovskites. Sci. Rep. 2016, 6, 28618.

82. Fraccarollo, A.; Cantatore, V.; Boschetto, G.; Marchese, L.; Cossi, M. Ab initio modeling of 2D layered organohalide lead perovskites. J. Chem. Phys. 2016, 144, 164701.

83. Chuang, S.-L.; Schmitt-Rink, S.; Miller, D. A. B.; Chemla, D. S. Exciton Green's-function approach to optical absorption in a quantum well with an applied electric field. Phys. Rev. B 1991, 43, 1500-1509. 